\title{
BIOSORCIÓN DE Cd, Pb y Zn POR BIOMASA PRETRATADA DE ALGAS ROJAS, CÁSCARA DE NARANJA Y TUNA
}

\section{BIOSORPTION OF Cd, Pb AND Zn BY PRETREATED BIOMASS RED ALGAE, ORANGE PEEL AND TUNA}

\author{
Lissette Vizcaíno Mendoza', Natalia Fuentes Molina² \\ Fecha de recepción: 5 de Septiembre de 2014 \\ Fecha de aprobación: 18 de Diciembre de 2014
}

Referencia: L. Vizcaíno Mendoza, N. Fuentes Molina (2015). Biosorción de Cd, Pb y Zn por biomasa pretartada de algas rojas, cáscara de naranja y tuna. Ciencia e Ingeniería Neogranadina, 25 (1), pp. 43 - 60

\section{RESUMEN}

Debido a su movilidad en los ecosistemas y a su toxicidad para las formas superiores de vida, los metales pesados $\mathrm{Cd}$, Pb y Zn son priorizados como unos de los contaminantes inorgánicos más importantes debido al alto riesgo que representan para el medio ambiente. Con el objeto de disminuir su concentración se diseñó un sistema para evaluar su remoción empleando biomasa de algas rojas, cáscaras de naranja (Citrus sp.) y tuna guajira (Opuntia sp.). Se estudió la influencia del pretratamiento y el empaquetamiento mediante ensayos tipo batch, en los que se emplearon soluciones de sodio y calcio. Se obtuvo como resultado una mayor capacidad de sorción de las algas modificadas con $\mathrm{NaOH} 0.1 \mathrm{~N}$ y de la naranja y la tuna con modificación sucesiva con $\mathrm{NaOH}$ y $\mathrm{CaCl}_{2} 0.2 \mathrm{M}$, y una afección poco significativa $(\leq 1 \%)$ del proceso de sorción al empacar la biomasa el placas planas de tul poliéster. La eficiencia de remoción se determinó mediante un reactor de flujo continuo de columna fija con un volumen líquido de $400 \mathrm{~mL}, 75 \mathrm{~g}$ de biomasa y tiempos de retención promedio de 1 y 2 h. Los resultados mostraron una eficiencia similar de las tres biomasas para remover $\mathrm{Cd}$ y $\mathrm{Pb}$, con promedios superiores al 95\%, mientras que el Zn se removió con mejor eficiencia (62\%) al emplear tuna modificada como sorbente. Finalmente, el material se calcinó a $700{ }^{\circ} \mathrm{C}$ con lo que se obtuvo una ceniza estable frente a soluciones ácidas, lo cual garantiza la captura de los metales removidos.

Palabras clave: Biosorción, Cadmio (Cd), Plomo (Pb), Zinc (Zn), alga roja, cáscara de naranja, tuna guajira.

1. Ing. Medio Ambiente, Docente Facultad de Ingeniería, Investigador Grupo Madre Tierra. Universidad de La Guajira, Fonseca, La Guajira, Colombia, Ivizcaino@uniguajira.edu.co

2. M.Sc. Ciencias Ambientales, Docente Facultad de Ingeniería, Investigador Grupo Madre Tierra. Universidad de La Guajira, Fonseca, La Guajira, Colombia,nnfuentes@uniguajira.edu.co 


\section{ABSTRACT}

Because of their mobility in ecosystems and their toxicity to higher forms of life, heavy metals $\mathrm{Cd}, \mathrm{Pb}$ and $\mathrm{Zn}$ are identified as some of the most important inorganic contaminants due to the high risk they pose to the environment. In order to decrease their concentration, a system to evaluate the removal of these metals using biomass of red algae, orange peel (Citrus sp.) and tuna Guajira (Opuntia sp.) was designed. The influence of pretreatment and packaging was studied through batch type tests using solutions of sodium and calcium. The results showed greater sorption capacity of $0.1 \mathrm{~N} \mathrm{NaOH}$ modified algae and of orange and tuna with $\mathrm{NaOH}$ and $\mathrm{CaCl}_{2} 0.2 \mathrm{M}$ subsequent modification, as well as a minimal effect $(\leq 1 \%)$ on the sorption process when packing biomass in tulle polyester flat plates. The removal efficiency was measured with a fixed-bed continuous flow reactor, with a liquid volume of $400 \mathrm{~mL}, 75 \mathrm{~g}$ of biomass, and average retention times of 1 to $2 \mathrm{~h}$. The results showed similar efficiency of the three types of biomasses to remove $\mathrm{Cd}$ and $\mathrm{Pb}$, with an average greater than $95 \%$, while $\mathrm{Zn}$ was removed with improved efficiency (62\%) using modified tuna as a sorbent. Finally, the material was charred at $700{ }^{\circ} \mathrm{C}$ resulting in an ash stable against acid solutions ( $\mathrm{pH} 2.5$ to 4.5), which ensures the capture of the removed metals.

Keywords: Biosorption, Cadmium (Cd), Lead (Pb), Zinc (Zn), red algae, orange peel, tuna Guajira.

\section{INTRODUCCIÓN}

La búsqueda de nuevos materiales y técnicas utilizables como alternativa para el tratamiento de aguas residuales provenientes de procesos industriales y de minería se ha constituido como un desafío de los últimos tiempos [1].

Estudios recientes han establecido el uso de metodologías alternativas para la adsorción de contaminantes, tales como los metales pesados, que emplean materiales de origen biológico como bacterias, algas y hongos, residuos industriales, agrícolas y urbanos, debido a su gran viabilidad, bajo costo y alta eficiencia de remoción. Dentro de este amplio conjunto de materiales biológicos se destaca la aplicación de las cáscaras de frutas cítricas (naranja) [1, 3, 4], algas marinas [5] y el nopal o tuna [6].
Una de las técnicas empleadas para dichos procesos es la biosorción, que consiste en la transferencia selectiva de uno o más solutos de una fase líquida a un lote de partículas sólidas de material biológico e involucra la participación de diversos mecanismos físicos y químicos en función de diversos factores [78]. Debido al origen natural de los sustratos y a la eliminación de lodos residuales durante el proceso de remoción, esta alternativa tecnológica se constituye en un sistema que permite no sólo remover el metal contaminante disminuyendo el impacto ambiental generado sobre el medio en el cual se descarga, sino que además permite recuperarlo para integrarlo a un nuevo ciclo productivo.

Sin embargo, su aplicación industrial, ambiental y/o sanitaria generalmente está limitada por la inestabilidad química y estructural de algunos 
materiales utilizados como biosorbentes [4]. Por esta razón, es necesario realizar ensayos de laboratorio y/o a escala que permitan evaluar la eficiencia de remoción de las diversas biomasas de uso potencial y de los sistemas a través de los cuales se realizan los ensayos de biosorción, para simular las características de los efluentes en condiciones ambientales y establecer los rangos óptimos en los cuales la biomasa es efectiva en cada uno de los parámetros que intervienen en el proceso.

La presente investigación ha considerado el estudio del potencial biosorbente de biomasa de algas rojas, cáscara de naranja y tuna guajira como una alternativa para lograr la remoción de los metales pesados presentes en los drenajes ácidos generados por la explotación minera de carbón desarrollada en los departamentos del Cesar y La Guajira. Como iones de análisis y estudio se estableció la utilización de los metales Plomo, Cadmio y Zinc, los cuales según Volesky [9] representan un alto riesgo para la salud, el ambiente y las reservas de recursos naturales, razón por la cual su estudio y eliminación de los ecosistemas reviste un interés prioritario frente a otros metales presentes en efluentes residuales.

\section{MATERIALES Y MÉTODOS}

A fin de determinar la presencia de los metales $\mathrm{Cd}, \mathrm{Pb}$ y $\mathrm{Zn}$ y sus respectivas concentraciones en los efluentes de minería se analizaron cuatro muestras de efluentes residuales derivados del proceso de extracción de carbón y almacenamiento de material estéril del área carbonífera ubicada en el sur del departamento de La Guajira. Con base en los valores obtenidos se prepararon las soluciones sintéticas multimetálicas empleadas en los diferentes ensayos realizados.
La fase experimental de la investigación se desarrolló en tres etapas que se resumen en la Figura 1. Todos los ensayos definidos para cada etapa se realizaron por duplicado.

\subsection{ETAPA 1. PREPARACIÓN DE LA BIOMASA Y LA SOLUCIÓN DE TRABAJO}

Los especímenes de algas marinas rojas tanto flotantes como adheridos al fondo marino (a $200 \mathrm{~m}$ de la línea de costa) se recolectaron en la playa Valle de los Cangrejos del municipio de Riohacha en el caribe colombiano; se lavaron con agua de la llave para eliminar residuos de arena y otros materiales marinos; y se almacenaron bajo refrigeración para garantizar su conservación [5]. Las cáscaras de naranja (Citrus sp) se obtuvieron en cafeterías y restaurantes ubicados en la zona comercial del municipio de Fonseca, La Guajira. Por su parte, las tunas (Opuntia Sp.) se recolectaron en el corregimiento de Conejo, zona rural del municipio de Fonseca. Una vez en el laboratorio, las biomasas se cortaron en pequeños trozos y se lavaron un par de veces con agua de la llave para eliminar compuestos solubles adheridos a éstas, que pudiesen estar presentes en el material, capaces de interferir en el desarrollo de los ensayos [10]. Posteriormente, se lavaron con agua destilada a $40{ }^{\circ} \mathrm{C}$ para eliminar aceites esenciales y diferentes impurezas, se secaron en una estufa a $60{ }^{\circ} \mathrm{C}[2,6,11]$ hasta obtener un peso constante, se trituraron y se almacenaron para su posterior uso.

Las soluciones de trabajo o drenajes ácidos de mina de laboratorio (DAMLab) se prepararon empleando agua desionizada, purificada por ósmosis inversa mediante equipo Desionizador Thermo Scientific Barnstead /Easypurell RF y utilizando como reactivos para la obtención de cada metal los siguientes compuestos: óxido 


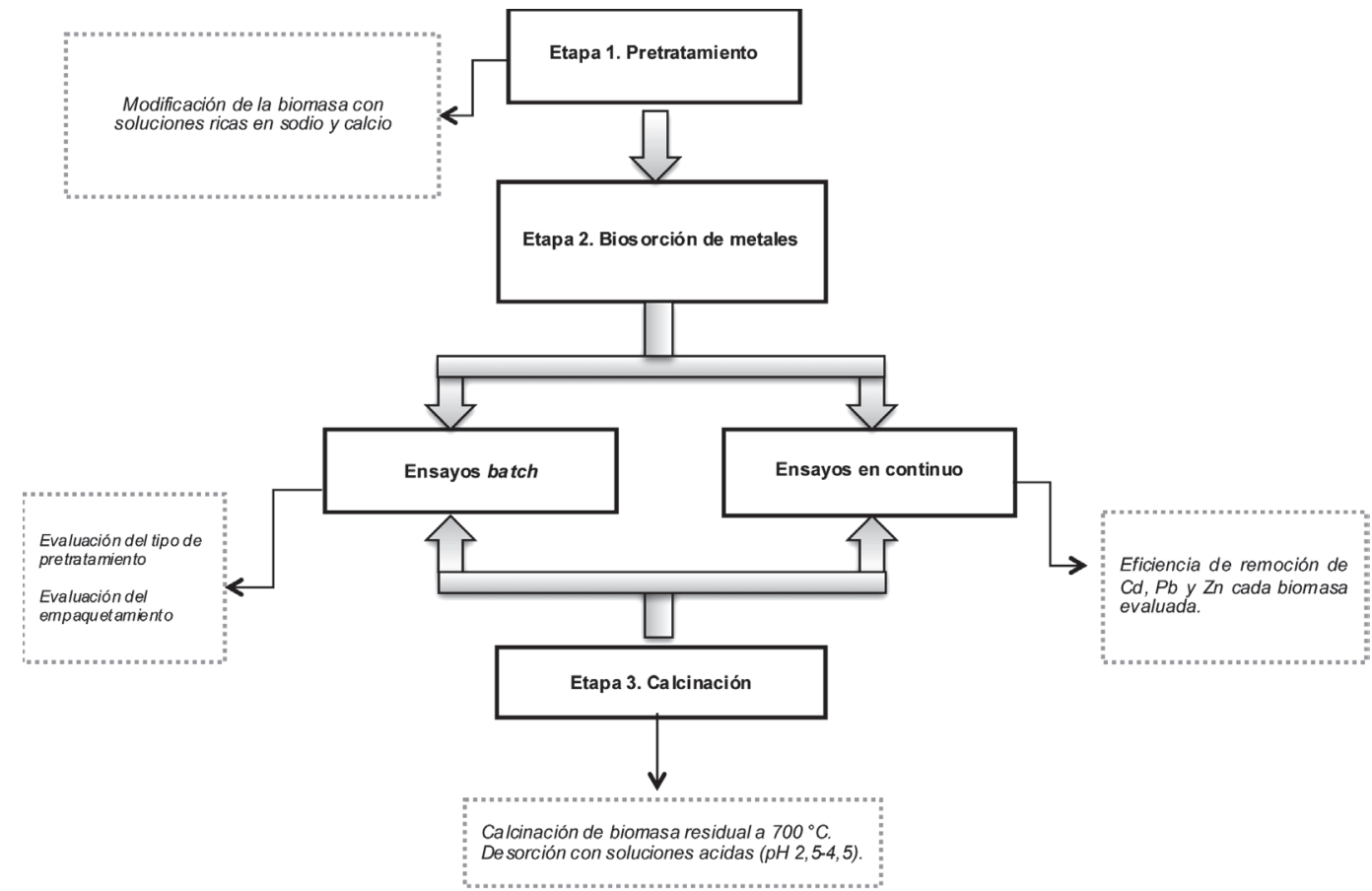

Figura 1. Etapas de la fase experimental de sorción de Cd, Pb y Zn con biomasa inerte de algas rojas, Citrus sp. y Opuntia sp.

de plomo en polvo (PbO: 98\%), cloruro de zinc granular $\left(\mathrm{ZnCl}_{2}: 95 \%\right)$ y cadmio metálico (Cd: 95\%). Para ajustar el valor del pH se utilizó ácido clorhídrico (95\%) e hidróxido de sodio $(95 \%)$ a concentración $1 \mathrm{M}$. Todos los reactivos empleados fueron de grado analítico.

\subsection{ETAPA 2. BIOSORCIÓN DE PB, CD Y ZN EN PROCESO BATCH}

Estas pruebas se realizaron con el objetivo de estudiar la influencia generada por la modificación de variables, tales como el pretratamiento de las biomasas con diferentes soluciones químicas y el empaquetamiento de las mismas sobre la remoción de los metales. Los experimentos se realizaron en proceso batch, empleando $100 \mathrm{~mL}$ de DAM y empleando 1.4 gramos de biomasa triturada, equivalente a una relación de $50 \mathrm{~g}$ biomasa/L DAM $_{\text {lab }}$ [5]. El sistema se agitó a 200 rpm durante 180 min. Mediante filtrado se separó el biomaterial de la solución. Finalmente, se midió el pH y la concentración de metales en la solución resultante. Todos los experimentos en proceso batch se hicieron por duplicado y a temperatura ambiente (aproximadamente 28 $\left.{ }^{\circ} \mathrm{C}\right)$, según los siguientes procedimientos:

\subsubsection{Estudio del pretratamiento}

Con el objetivo de comprobar si algunos constituyentes de los biomateriales influyen en la remoción de los metales se hicieron prelavados con diversas soluciones definidas con base en su amplia utilización en procesos de biosorción con materiales de características biológicas iguales o similares a los empleados en la presente investigación. Se prepararon soluciones de $\mathrm{NaOH}$ 
y Ca $(\mathrm{OH})_{2}$, cada una con una concentración de $0.1 \mathrm{~N}$ en el caso de las algas $[5,12]$, y soluciones de $\mathrm{NaOH}$ y $\mathrm{CaCl}_{2}$ a concentración de $0.2 \mathrm{M}$ para las naranjas y las tunas $[1,6,13]$.

En ensayos por separado, cada solución fue agregada al alga en una relación de $10 \mathrm{~g}$ de biomasa por $1 \mathrm{~L}$ de disolución durante $12 \mathrm{~h}$. Luego se lavaron con agua destilada a temperatura ambiente para eliminar el exceso de $\mathrm{Na}$ y Ca y se secaron a $60^{\circ} \mathrm{C}$ hasta peso constante. Por su parte, las cáscaras de naranja y tuna inicialmente fueron tratadas empleando $30 \mathrm{~g}$ de biomasa seca y tratada de malla 180 - $250 \mu$ m en 500 ml de una solución de $\mathrm{NaOH} 0.2 \mathrm{M}$ a una temperatura de $4^{\circ} \mathrm{C}$, manteniendo una agitación constante por $2 \mathrm{~h}$ sin alterar la temperatura. Luego se dejaron reposar y se filtraron haciendo lavados sucesivos con agua destilada para eliminar el exceso de $\mathrm{NaOH}$, después se secaron en una estufa a una temperatura de $60^{\circ} \mathrm{C}[3,13]$. Posteriormente se tomaron 20 gramos de biomasa pretratada con $\mathrm{NaOH}$, se colocaron en $500 \mathrm{ml}$ de una solución de $\mathrm{CaCl}_{2} 0.2 \mathrm{M}$, manteniendo la mezcla en agitación constante de 200 rpm durante 24 h [1, 3].

Los diferentes tipos de biomateriales pretratados se colocaron en contacto con el DAM $_{\text {Lab }}$ en las condiciones de operación antes descritas, empleando $1.4 \mathrm{~g}$ de biomasa y 100 $\mathrm{mL}$ de solución, equivalente a una relación de $14 \mathrm{~g}$ biomasa/L drenaje, 200 rpm y 180 minutos de contacto [5]. El pH se ajustó a 4.5 con disoluciones de $\mathrm{NaOH}$ o $\mathrm{HCl}(1 \mathrm{M})$. Los ensayos se realizaron a temperatura ambiente.

\subsubsection{Estudio del tipo de empaque}

Teniendo en cuenta los volúmenes de drenaje ácido generados en las minas de carbón, así como las condiciones de funcionamiento de éstas, un diseño apropiado para la descontaminación del drenaje es un reactor que opere en continuo, que incluya un sistema que permita la remoción y recambio permanente del material biológico. Con el objeto de facilitar la manipulación del lecho biológico del reactor, se realizaron ensayos preliminares en los cuales se evaluó el efecto de empacar la biomasa sobre el proceso de adsorción de los metales. Para tal fin se emplearon vasos de precipitado con $100 \mathrm{~mL}$ de solución sintética que, en ensayos por separado, se colocaron en contacto durante 120 min y a temperatura ambiente con $5 \mathrm{~g}$ de biomasa suelta $\mathrm{y}$ empacada en módulos planos circulares de tul poliéster de $7 \mathrm{~cm}$ de diámetro [5].

\subsection{ETAPA 3. COLUMNA DE FLUJO CONTINUO PARA ADSORCION DE PLOMO, CADMIO Y ZINC}

El sistema más empleado para el estudio de biosorción en continuo ha sido el de lecho fijo. En este, las partículas del biosorbente permiten el paso del fluido de forma tortuosa sin separarse unas de otras, haciendo que la altura del lecho y, en consecuencia, su porosidad se mantengan constantes. La saturación de la columna es controlada por parámetros como el tiempo, el espacio y el tamaño de la columna, [9]. Este sistema resulta ser el más efectivo para la remoción de metales pesados $[14,15]$.

La columna de lecho empacado con flujo ascendente se construyó manualmente en el laboratorio utilizando un cilindro graduado con diámetro de $6.5 \mathrm{~cm}$ y $42.5 \mathrm{~cm}$ de longitud. En la parte inferior se acondicionó una entrada para el afluente sintético con contenido de $\mathrm{Cd}$, Pb y Zn, mientras que en la parte superior se ubicó una salida lateral para el efluente tratado. En la columna se colocaron 75 g de la biomasa pretratada definida en los ensayos 
batch, previamente empaquetada en malla de tul poliéster, formando módulos de cinco empaques con diámetro de $5 \mathrm{~cm}$ confinados en cilindros (debidamente perforados para garantizar el contacto de la biomasa con la solución) de $10.3 \mathrm{~cm}$ de longitud y $5.5 \mathrm{~cm}$ de diámetro, sellados en sus extremos con malla de tul poliéster, para una relación longitud/ diámetro de 6.2. La alimentación del DAM $\mathrm{Dab}_{\text {La }}$ se hizo empleando una bomba peristáltica Pulsafeeder modelo Pulsatron serie Aplus 3919/2010, mientras que la salida del drenaje tratado se hizo por rebose. Para estudiar la evolución del proceso de remoción de metales en la unidad A se realizó un muestreo periódico: cada hora, durante 120 min, en ensayos por duplicado se determinaron las concentraciones de $\mathrm{Pb}, \mathrm{Cd}$ y Zn en el efluente, así como el pH final de la solución [5]. En la Figura 2 se muestra el esquema del sistema empleado.

Antes de pasar la solución metálica a través de la columna de adsorción, el material fue humedecido con agua destilada a fin de evitar una disminución repentina de la concentración del metal en la solución de salida, debido a una rápida adsorción del metal por la biomasa seca, sobre todo en el primer momento en que hace contacto la biomasa con la solución [16].

El volumen del efluente tratado se estudió como una variable dependiente del tiempo de residencia y la relación longitud/diámetro, según el modelo empírico propuesto por Díaz et al. [5] de acuerdo con la siguiente expresión matemática. El modelo es apto para tiempos de residencia de 1 a 2 h y relación longitud/diámetro de 1.5 a 11.6.

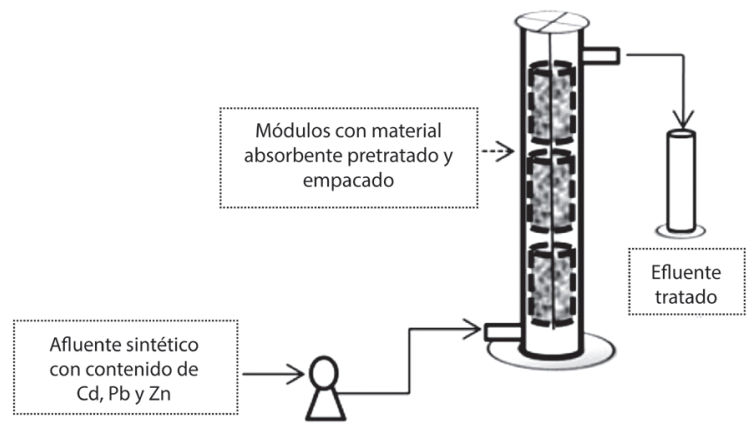

Figura 2. Esquema de la columna de lecho empacado fijo con flujo ascendente para el proceso de biosorción con algas rojas, cáscara de naranja y tuna.

Las variables operativas de funcionamiento del reactor se muestran en la Tabla 1. Los caudales empleados son similares a los ensayados por Agoubourde [17], quien empleó borras de salmuera y aserrín para remoción de Zn y Cu, y González [18], quién empleó desechos agrícolas y de crustáceos para remoción de Cu, Cd y Cr.

Tabla 1. Tiempos de retención, caudales de trabajo y velocidades de flujo empleados en la realización de los ensayos en continuo.

\begin{tabular}{|c|c|c|c|c|}
\hline BIOMATERIAL & Ensayo & $\begin{array}{c}\text { Tr } \\
\text { (min) }\end{array}$ & $\begin{array}{c}\text { Caudal } \\
\text { Prom. } \\
\text { (mL/ } \\
\text { min) }\end{array}$ & $\begin{array}{c}\text { Volumen } \\
\text { tratado } \\
\text { (mL) }\end{array}$ \\
\hline A1 & ACT1 & 64,19 & 1,56 & 55,03 \\
& ACT2 & 112,4 & 0,89 & 94,03 \\
\hline N2 & NCT1 & 64,27 & 1,56 & 55,09 \\
\hline T2 & NCT2 & 123,25 & 0,81 & 102,81 \\
\hline TCT1 & 79,1 & 1,26 & 67,09 \\
1072 & 128,6 & 0,78 & 107,14 \\
\hline
\end{tabular}

ACT1, NCT1 y TCT1 (ensayos en continuo con alga, naranja y tuna a tiempo de contacto 1), ACT2, NCT2 y TCT2 (ensayos en continuo con alga, naranja y tuna a tiempo de contacto 2), Tr tiempo de retención.

$$
\left.V=\left[\frac{0,3762 L}{D}+1,8525 \operatorname{Tr}\right]-\left[\left(\frac{0,1683 L}{D} * \operatorname{Tr}\right)-0,7644\right)\right]
$$

Donde $V$ es el volumen de efluente tratado, L es la longitud del lecho en la columna, D es el diámetro de la columna y $\mathrm{Tr}$ es el tiempo de residencia. 


\subsection{ETAPA 4. CALCINACION DE BIOMASA RESIDUAL}

Los estudios de desorción en lote son importantes para el diseño de un proceso de biosorción, ya que pueden permitir el reciclaje tanto del adsorbente como del adsorbato, contribuyendo a minimizar los volúmenes de desechos generados y a reducir los costos del proceso [15]. Sin embargo, cuando la biomasa se satura y se convierte en un residuo peligroso se hace necesaria su inactivación. En este sentido, la calcinación por vía seca se muestra como una alternativa para su manejo, debido a que es un proceso que permite encapsular los metales en la matriz biológica y evita que estos se liberen generando un nuevo problema de contaminación, al mismo tiempo que reduce el volumen y la masa de material residual [19]. Por su parte, las cenizas remanentes del proceso pueden ser nuevamente incluidas en el ciclo de tratamiento debido a su potencial como adsorbente [20], de metales tales como Ag y Cu [21], y $\mathrm{Cr}$ [22].

Con el objetivo de determinar la capacidad de encapsulamiento de cada biomaterial se realizaron ensayos en los cuales la biomasa remanente de los procesos de adsorción en flujo continuo se calcinó a 600 y $700{ }^{\circ} \mathrm{C}$ en una mufla durante $1 \mathrm{~h}$ hasta convertirse en cenizas. Posteriormente se realizaron ensayos en los que se emplearon $1 \mathrm{~g}$ de ceniza y $100 \mathrm{~mL}$ de solución ácida de $\mathrm{pH} 2.5$ y 4.5, preparada con ácido nítrico [15] al 75\%, durante un periodo de $24 \mathrm{~h}$. Las disoluciones resultantes se filtraron y se procedió a la determinación de la concentración de cada metal en el efluente acido resultante a fin de establecer el porcentaje de metal desorbido y el pH final de la solución [5].

\section{RESULTADOS Y ANÁLISIS}

\subsection{ETAPA 1. PREPARACIÓN DE LA BIOMASA Y LA SOLUCIÓN DE TRABAJO}

Se analizaron cuatro muestras de efluentes residuales derivados del proceso de extracción de carbón y almacenamiento de material estéril del área de estudio y se obtuvieron los resultados contenidos en la Tabla 2. A partir de los valores de $\mathrm{pH}$ determinados en el análisis físico químico de las muestras tomadas y basados en la clasificación establecida por Skousen and Ziemkiewics [23] se determinó que los efluentes derivados del proceso de minería tienen características ácidas (drenajes tipo I: $\mathrm{pH}<4,5$ y II: $\mathrm{pH}<6,0$ ) con alto potencial de solubilización derivado de las concentraciones de iones metálicos ( $C d, P b$ y $Z n$ ) en solución acuosa, cuya presencia es consecuencia de los procesos de oxidación de sulfuros que suceden entre la roca que queda al descubierto y/o el material extraído y el ambiente [24]. En cuanto a las concentraciones de cada ion, se observa una gran diferencia entre los valores de Cd y las otras dos especies químicas evaluadas, lo cual según Dionisio [25] es lógico debido a que la presencia de dicho metal en el ambiente depende directamente del contenido de $\mathrm{Zn}$ y Pb, metales a los cuales generalmente se halla asociado en forma de sulfuros en porcentajes cercanos al $1 \%$.

Con base en los resultados anteriores se definieron las concentraciones de trabajo para preparar las soluciones en el laboratorio. Se tomaron en cuenta sólo las concentraciones reportadas por las muestras 1 y 2 por el hecho de que estas reportaron las concentraciones más altas de Cd, a fin de evitar que las concentraciones finales reportaran valores por debajo del Límite de Detección de Cd del equipo de absorción (0.014 mg Cd /L) dificultando el cálculo de los porcentajes de remoción. 
Tabla 2. Caracterización físico química de los drenajes ácidos de mina de la zona sur del departamento de La Guajira.

\begin{tabular}{|c|c|c|c|c|}
\hline Parámetro & DAM 1 & DAM 2 & DAM 3 & DAM 4 \\
\hline $\mathrm{pH}$ & 3,55 & 4,38 & 5,23 & 4,93 \\
\hline Conductividad $(\mu \mathrm{s} / \mathrm{cm})$ & 1040 & 577 & 393 & 818 \\
\hline Cadmio $(\mathrm{mg} / \mathrm{L})$ & 5,951 & 1,33 & 0,796 & 0,234 \\
\hline Plomo $(\mathrm{mg} / \mathrm{L})$ & 23,573 & 4,37 & 5,87 & 2,18 \\
\hline Zinc $(\mathrm{mg} / \mathrm{L})$ & 58,0 & 38,0 & 0,353 & 48,05 \\
\hline
\end{tabular}

DAM: Drenaje acido de mina en las muestras 1, 2, 3 y 4.

Para el análisis de influencia de pretratamiento y ensayos en columna se optó por emplear las concentraciones más altas analizadas (DAM1), a fin de facilitar el estudio del comportamiento de las biomasas frente a la presencia de las concentraciones más altas medidas de $\mathrm{Cd}, \mathrm{Pb}$ y $\mathrm{Zn}$. Por su parte, los ensayos de empaquetamiento se realizaron empleando los valores medidos para cada metal en el DAM2. Este cambio en los valores de la solución se justifica debido a que en dichas pruebas lo fundamental es determinar la influencia del empaque sobre el porcentaje de remoción del metal asociado a cada biomasa independientemente de su concentración inicial, con lo cual, si las concentraciones disminuyen, no se generan alteraciones en la prueba y adicionalmente se minimiza la toxicidad de la solución residual.

\subsection{ETAPA 2. BIOSORCIÓN DE METALES EN PROCESO BATCH}

\subsubsection{Efecto del pretratamiento del biomaterial}

Los resultados de los ensayos se muestran en la Tabla 3. Las concentraciones finales más bajas para Cd y Zn fueron registradas por T2, mientras que N2 reporto la concentración más baja de Pb.
Las tres biomasas reportaron eficiencias de remoción de Cd superiores al 99\%, mientras que para $\mathrm{Pb}$ los mejores porcentajes fueron reportados por A1 (97\%), N2 (99\%) y T2 (96\%). De acuerdo con los resultados se puede afirmar que el pretratamiento solo es necesario para incrementar la capacidad de captación de Zn. Sin embargo, debido a que en condiciones reales los efluentes contaminados con metales generalmente presentan una gran variedad de iones disueltos, resulta necesario pretratar todas las biomasas para lograr una remoción eficiente de todos los metales evaluados en una misma solución.

A excepción de los resultados para $\mathrm{Pb}$ del ensayo T1 (remoción del $81 \%$, lo cual se trata de una curiosidad no fundamentada), la remoción de $\mathrm{Cd}^{+2}$ y $\mathrm{Pb}^{+2}$ reportada por los biosorbentes (independientemente del pretratamiento) supera el $91 \%$. En el caso del Zn, se observa una mayor eficiencia con la biomasa pretratada al obtener eficiencias máximas superiores al 70\% para las algas y al $80 \%$ para la naranja y tuna, lo cual sugiere que la tuna tiene una afinidad ligeramente mayor por los iones de $\mathrm{Zn}^{+2}$ que las otras dos biomasas. En la Figura 2 se presenta el comportamiento de la biosorción de metales pesados expresado en función de las concentraciones finales obtenidas empleando 
Tabla 3. Influencia del pretratamiento sobre la eficiencia de biosorción de cadmio, plomo y zinc.

\begin{tabular}{|c|c|c|c|c|c|c|c|c|c|c|}
\hline \multirow{2}{*}{$\begin{array}{l}\text { Tipo de } \\
\text { biomaterial }\end{array}$} & \multirow{2}{*}{$\begin{array}{l}\text { Tipo de } \\
\text { pretratamiento }\end{array}$} & \multicolumn{3}{|c|}{$\begin{array}{c}\text { Concentraciones } \\
\text { (mg/l) }\end{array}$} & \multicolumn{3}{|c|}{ q (mg/g) } & \multicolumn{3}{|c|}{ \% Remoción } \\
\hline & & Cd & $\mathrm{Pb}$ & $\mathrm{Zn}$ & Cd & $\mathrm{Pb}$ & $\mathrm{Zn}$ & Cd & $\mathbf{P b}$ & $\mathrm{Zn}$ \\
\hline \multirow{3}{*}{ Algas Rojas } & A1 & 0,55 & 2,2 & 22,45 & 0,386 & 1,527 & 2,539 & 99,3 & 97,3 & 71,9 \\
\hline & A2 & 0,46 & 3 & 33,69 & 0,392 & 1,470 & 1,736 & 99,4 & 96,3 & 57,9 \\
\hline & АЗ & 0,93 & 7,1 & 41,88 & 0,359 & 1,177 & 1,151 & 98,8 & 91,1 & 47,7 \\
\hline \multirow{3}{*}{ Cáscara de naranja } & N1 & 0,47 & 3,2 & 29,35 & 0,392 & 1,455 & 2,046 & 99,4 & 96 & 63,3 \\
\hline & N2 & 0,29 & 0,26 & 15,03 & 0,404 & 1,665 & 3,069 & 99,6 & $>99$ & 81,2 \\
\hline & N3 & 0,44 & 1,6 & 47,94 & 0,394 & 1,570 & 0,719 & 99,5 & 98 & 40,1 \\
\hline \multirow{3}{*}{ Tuna } & T1 & 0,4 & 15 & 37,4 & 0,397 & 0,612 & 1,471 & 99,5 & 81,3 & 53,3 \\
\hline & T2 & 0,26 & 5,8 & 13,03 & 0,407 & 1,270 & 3,212 & 99,7 & 92,8 & 83,7 \\
\hline & T3 & 0,63 & 3,3 & 48,53 & 0,380 & 1,448 & 0,676 & 99,2 & 95,9 & 39,3 \\
\hline
\end{tabular}

A1 (alga tratada con $\mathrm{NaOH}$ ), A2 (alga tratada con $\mathrm{Ca}(\mathrm{OH})$ ), N1 y T1 (naranja y tuna tratada con NaOH) N2 y T2 (naranja y tuna tratada con $\mathrm{NaOH}$ y $\mathrm{CaCl}_{2}$ ), A3, $\mathbf{N 3}$ y T3 (alga, naranja y tuna natural).

biomasa modificada y en su estado natural. En la gráfica no se observan cambios importantes en la distribución de los datos obtenidos en cada análisis de acuerdo al tipo de pretratamiento.

Tal y como se observó en los ensayos realizados por Rodríguez et al. [26] para remoción de $\mathrm{Pb}^{2+}$, $\mathrm{Cd}^{2+}$ y $\mathrm{Cr}^{3+}$ empleando Saccharomyces cerevisiae no-viva, el tratamiento previo tuvo un efecto significativo sobre la biosorción de iones de $\mathrm{Pb}$ y $\mathrm{Zn}$, mientras que para la biosorción del $\mathrm{Cd}^{2+}$ son notables los efectos de la concentración de biomasa. Esto explica el hecho de que la remoción de Cd se mantuviera constante en los ensayos de pretratamiento al lograr una eficiencia de remoción superior al 99\% para todas las

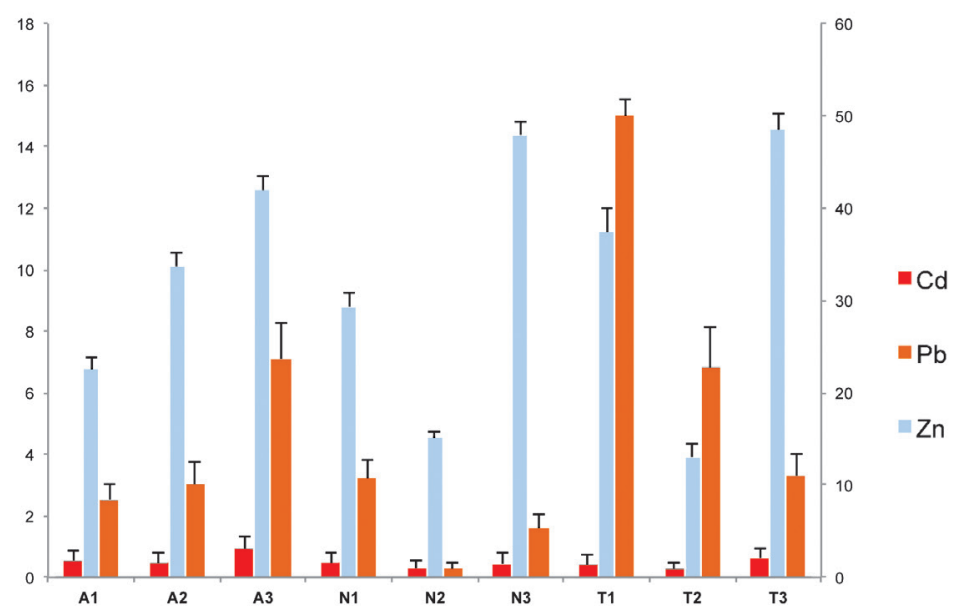

Figura 2. Concentraciones finales de Cd, Pb y Zn obtenidas al emplear biomasa natural y modificada de alga roja, cáscara de naranja y tuna. A1 (alga tratada con NaOH), A2 (alga tratada con Ca (OH)2), N1 y T1 (naranja y tuna tratada con $\mathrm{NaOH}$ ) N2 y T2 (naranja y tuna tratada con $\mathrm{NaOH}$ y $\mathrm{CaCl}_{2}$ ), $\mathbf{A 3}$, N3 y T3 (alga, naranja y tuna natural). 
biomasas en estudio, mientras que el $\mathrm{Pb}$ y el $\mathrm{Zn}$ variaron sus concentraciones finales de acuerdo con el tipo de solución química empleada.

Así mismo, a partir de los resultados se puede suponer una mayor afinidad de las biomasas por los iones de $\mathrm{Pb}$ y $\mathrm{Cd}$ y una mejor relación de dichos metales con los sitios activos presentes en la superficie de las matrices vegetales analizadas [27]. Sin embargo, para corrobar esta afirmación es necesario evaluar la cinética del proceso y determinar las respectivas isotermas de sorción para cada metal y/o biomasa ensayada [5, 28]. En el caso del Zn, el porcentaje de remoción es más bajo como consecuencia de una menor capacidad de intercambio de los radicales libres en la superficie del biosorbente con el metal o por interferencias causadas por los otros dos metales presentes en la solución.

Las biomasas de algas rojas, cáscara de naranja y tuna presentaron una mejor capacidad de captación de Cd en ausencia de pretratamiento y a pH 4.5 (99\% de remoción) que la biomasa pretratada de Musa paradisiaca L. $190 \%$ de remoción) empleada en soluciones con valor de $\mathrm{pH}$ de 6.0 unidades [29]. En contraste, se observó una menor remoción de Zn (cercana al $40 \%$ ) al emplear biomasa natural y un comportamiento ligeramente superior (70$80 \%$ ) al emplear biomasa modificada.

De igual manera, los resultados indican que las cáscaras de naranja (N2) y las tunas (T2) tienen una mayor capacidad que las algas rojas (A1) de remover en conjunto los tres metales en estudio disueltos en una misma solución.

2.2.2 Influencia del empaquetamiento sobre la eficiencia de sorción

Una vez determinados $A 1, N 2$ y $T 2$ como los biosorbentes con la mayor capacidad de remoción conjunta de $\mathrm{Cd}$, $\mathrm{Pb}$ y $\mathrm{Zn}$, se procedió a evaluar el efecto del empaquetamiento de las biomasas sobre la eficiencia de sorción de dichos iones realizando ensayos bajo las mismas condiciones anteriores. Los resultados se muestran en la Figura 3. Las concentraciones iniciales de $\mathrm{Cd}, \mathrm{Pb}$ y Zn fueron de $1.33 \mathrm{mg} / \mathrm{L}, 4.37 \mathrm{mg} / \mathrm{L}$ y 38.0 $\mathrm{mg} / \mathrm{L}$ respectivamente. Los resultados de los ensayos se presentan en las Tablas $4 a$ y 4b. Las diferencias entre los valores de concentraciones finales y porcentajes de remoción de la Tabla 3 respecto a las Tablas $4 a$ y $4 b$ se deben a que se emplearon diversas concentraciones iniciales para cada ensayo.

En la Figura 3 se muestran los resultados obtenidos en términos de las concentraciones finales frente a sus respectivos porcentajes de remoción.

Tal y como sucedió en los ensayos de pretratamiento, los iones $\mathrm{Cd}$ y $\mathrm{Pb}$ presentaron los porcentajes de remoción más altos. Así mismo, se observa una diferencia de $\pm 1 \%$ en los porcentajes de remoción de cada metal obtenidos con el sorbente en sus dos estados. Esta diferencia indica que el empaque no afecta considerablemente el fenómeno de biosorción y, adicionalmente, hace más práctica y funcional la manipulación del material biológico en los diferentes ensayos aplicados. Los resultados son similares a los obtenidos por Díaz et al. [5] empleando Sargassum sp. en condiciones parecidas.

La Figura 5 muestra los resultados obtenidos en los ensayos de flujo continuo. Los porcentajes de remoción para $\mathrm{Cd}$ y Zn son superiores y/o cercanos a los reportados en ensayos que emplearon hongos de la podredumbre blanca (Cd 74\% y Zn 57\%) [19], y Eleocharis acicularis (Cd 80\% y Zn 75\%) [27]. Así 


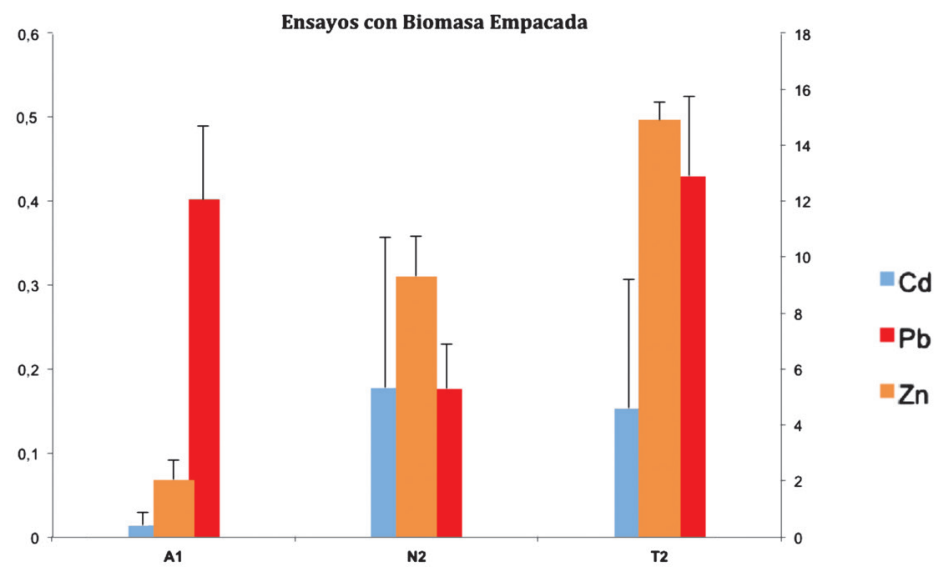

Figura 3. Concentraciones finales de Cd, Pb y Zn en ensayos con biomasa suelta y empacada. A1S (alga tratada con NaOH suelta), A1E (alga tratada con NaOH empacada), N2S y T2S (naranja y tuna tratada con NaOH y CaCl2 suelta), N2E y T2E (naranja y tuna tratada con $\mathrm{NaOH}$ y CaCl2 empacada).

Tabla 4a. Concentraciones finales, porcentajes de remoción y capacidad de adsorción de la biomasa suelta.

\begin{tabular}{|c|c|c|c|c|c|c|c|c|c|}
\hline \multirow{3}{*}{ Tipo de biomaterial } & \multicolumn{9}{|c|}{ Biomasa suelta } \\
\hline & \multicolumn{3}{|c|}{ CF (mg/L) } & \multicolumn{3}{|c|}{ \% Remociōn } & \multicolumn{3}{|c|}{$q(\mathrm{mg} / \mathrm{g})$} \\
\hline & Cd & $\mathrm{Pb}$ & $\mathrm{Zn}$ & Cd & $\mathrm{Pb}$ & $\mathrm{Zn}$ & Cd & $\mathrm{Pb}$ & Zn \\
\hline $\mathrm{A} 1$ & 0,024 & 0,372 & 2,37 & 98,2 & 91,5 & 93,8 & 0,0261 & 0,080 & 0,713 \\
\hline N2 & 0,165 & 0,26 & 8,961 & 87,6 & 94,1 & 76,4 & 0,0233 & 0,0822 & 0,581 \\
\hline $\mathrm{T} 2$ & 0,173 & 0,42 & 15,3 & 87 & 90,4 & 59,7 & 0,0231 & 0,079 & 0,454 \\
\hline
\end{tabular}

Tabla $4 \boldsymbol{b}$. Concentraciones finales, porcentajes de remoción y capacidad de adsorción de la biomasa empacada.

\begin{tabular}{|c|c|c|c|c|c|c|c|c|c|}
\hline \multirow{3}{*}{ Tipo de biomaterial } & \multicolumn{9}{|c|}{ Biomasa suelta } \\
\hline & \multicolumn{3}{|c|}{ CF (mg/L) } & \multicolumn{3}{|c|}{ \% Remoción } & \multicolumn{3}{|c|}{$q(\mathrm{mg} / \mathrm{g})$} \\
\hline & Cd & $\mathrm{Pb}$ & $\mathrm{Zn}$ & Cd & $\mathrm{Pb}$ & $\mathrm{Zn}$ & $\mathrm{Cd}$ & $\mathrm{Pb}$ & Zn \\
\hline $\mathrm{A} 1$ & 0,014 & 0,402 & 2,03 & 99 & 90,8 & 94,7 & 0,0263 & 0,0794 & 0,719 \\
\hline N2 & 0,178 & 0,176 & 9,32 & 86,6 & 96 & 75,5 & 0,0230 & 0,0839 & 0,574 \\
\hline $\mathrm{T} 2$ & 0,153 & 0,429 & 14,9 & 88,5 & 90,2 & 60,9 & 0,0235 & 0,0788 & 0,462 \\
\hline
\end{tabular}

A1 (alga tratada con NaOH), N2 y T2 (naranja y tuna tratada con NaOH y CaCl2). CF (concentración final)

mismo, la captación de iones fue menor a la reportada al emplear lodos activados $(98,73 \%$ $\mathrm{Zn}^{+2}$ ) [30], y a la reportada para el Pb $(99,5 \%)$ y Zn $(99,5 \%)$ al emplear cáscara de naranja modificada [4]. 


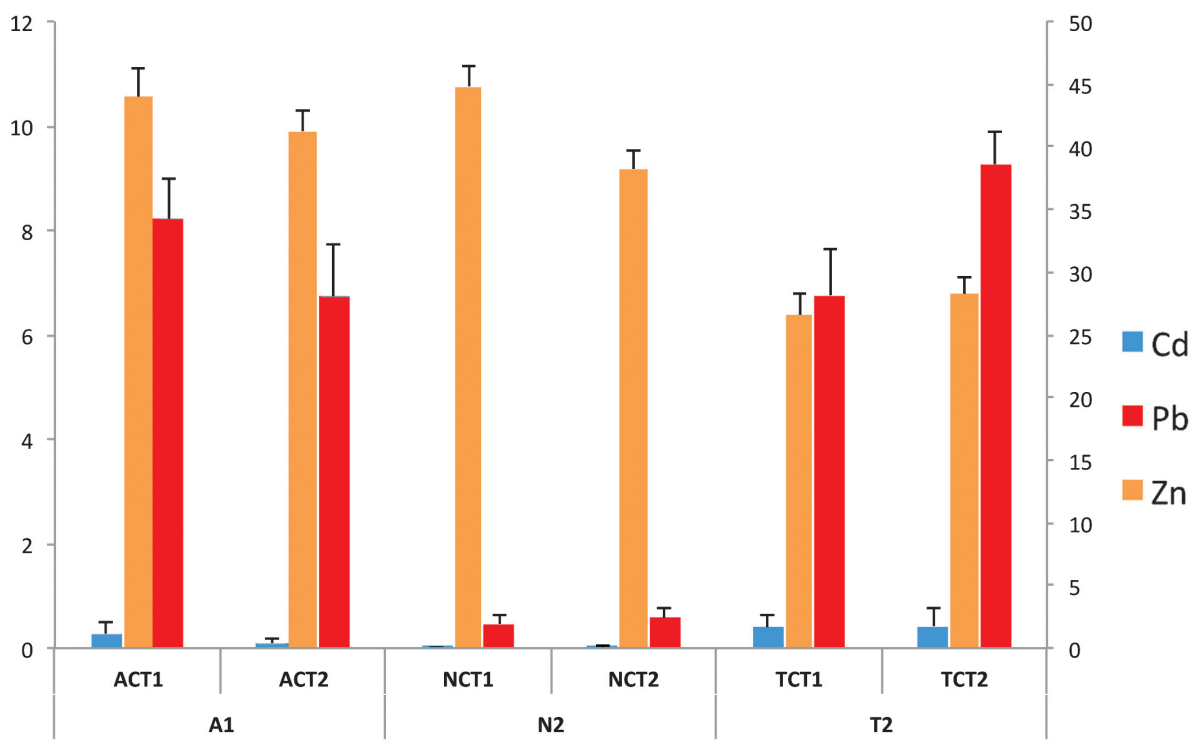

Figura 5. Concentraciones finales y porcentajes de remoción de Cd, Pb y Zn obtenidos en ensayos en continuo. ACT1 NCT1 y TCT1 (ensayos en continuo con alga, naranja y tuna a tiempo retención 1), ACT2, NCT2 y TCT2 (ensayos en continuo con alga, naranja y tuna a tiempo de retención 2).

Tabla 5. Remoción de Cd, Pb y Zn en ensayos continuos en reactores de columna fija.

\begin{tabular}{|c|c|c|c|c|c|c|c|c|c|c|c|}
\hline \multirow{2}{*}{ Biomaterial } & \multirow{2}{*}{ Ensayo } & \multicolumn{3}{|c|}{ Cf (mg/L) } & \multicolumn{3}{|c|}{ \% Remoción } & \multicolumn{3}{|c|}{$q(\mathrm{mg} / \mathrm{g})$} & \multirow{2}{*}{$\begin{array}{l}\mathrm{pH} \\
\text { final }\end{array}$} \\
\hline & & Cd & $\mathrm{Pb}$ & Zn & Cd & $\mathrm{Pb}$ & $\mathrm{Zn}$ & Cd & $\mathrm{Pb}$ & Zn & \\
\hline \multirow{2}{*}{ A1 } & ACT1 & 0,28 & 8,21 & 43,92 & 95,3 & 65,2 & 24,3 & 0,0302 & 0,0819 & 0,0751 & 6,22 \\
\hline & АСТ2 & 0,099 & 6,73 & 41,26 & 98,3 & 71,5 & 28,9 & 0,0312 & 0,0898 & 0,0893 & 7,03 \\
\hline \multirow{2}{*}{ N2 } & NCT1 & 0,014 & 0,47 & 43,83 & 99,8 & 98,0 & 24,4 & 0,0317 & 0,1232 & 0,0756 & 6,81 \\
\hline & NCT2 & 0,014 & 0,58 & 38,24 & 99,8 & 97,5 & 34,1 & 0,0317 & 0,1226 & 0,1054 & 7,00 \\
\hline \multirow{2}{*}{ T } & TCT1 & 0,392 & 6,76 & 26,61 & 93,4 & 71,3 & 54,1 & 0,0296 & 0,0897 & 0,1674 & 6,79 \\
\hline & TCT2 & 0,424 & 9,27 & 28,22 & 92,9 & 60,7 & 51,3 & 0,0295 & 0,0763 & 0,1588 & 7,36 \\
\hline
\end{tabular}

ACT1, NCT1 y TCT1 (ensayos en continuo con alga, naranja y tuna a tiempo de retención 1), ACT2, NCT2 y TCT2 (ensayos en continuo con alga, naranja y tuna a tiempo de retención 2).

En la Figura 5 se observa que la remoción de Cd al emplear alga roja y cáscara de naranja resultó ser muy similar, mientras que la tuna registró un valor ligeramente menor.
En el caso del $\mathrm{Pb}$, la naranja registró una mayor capacidad de adsorción que los otros dos biomateriales de estudio, mientras que la tuna mostró un comportamiento 
más eficiente en la remoción de Zn. Estos resultados sugieren que las biomasas presentan una mayor afinidad por los iones de $\mathrm{Cd}$ que por los de Zn. Esto se debe a que el radio iónico del Cd es mayor que el del $\mathrm{Zn}$; por tanto, éste es desplazado con mayor facilidad, permitiendo que el ion Cd pueda unirse mejor a los sitios activos sobre la superficie de la biomasa [31].

Así mismo, según Aguilar et al. [32] existe afinidad de la cáscara de naranja según la relación $\mathrm{Pb}>\mathrm{Cd}>\mathrm{Zn}$. De manera similar se comportaron las algas pardas Lessonia nigrescens y Macrocystis integrifolia empleadas por Cuizano et al. [33]. Este comportamiento se observó en los ensayos batch para análisis de empaquetamiento. Sin embargo, en los experimentos en columna las algas rojas, naranja y tunas mostraron una afinidad con tendencia $\mathrm{Cd}>\mathrm{Pb}>\mathrm{Zn}$.

Del mismo modo, se observaron resultados semejantes a los reportados por Navarro et al. [34] al emplear microorganismos, vegetales y algas naturales y modificadas comprobando la preferencia de los biosorbentes estudiados por el ion $\mathrm{Cd}^{+2}$ frente al ion $\mathrm{Zn}^{+2}$.

\subsection{ETAPA 4. CALCINACIÓN DE LA BIOMASA RESIDUAL}

Los ensayos de calcinación se realizaron tomando $10 \mathrm{~g}$ de la biomasa residual cargada de metales generada en los ensayos batch (pretratamiento) y de flujo continuo, en los que se obtuvieron los resultados descritos en la Tabla 6.

En la Figura 6 se observa que los biomateriales calcinados a $700{ }^{\circ} \mathrm{C}$ tienen la capacidad de retener el $99 \%$ del $\mathrm{Cd}$ adsorbido en todos los ensayos. Así mismo, se observa que para el caso del $\mathrm{Pb}$ y el $\mathrm{Zn}$, la ceniza retuvo de manera más eficiente los iones frente a la solución de mayor $\mathrm{pH}$, con un porcentaje promedio de desorción inferior al $2 \%$. En los ensayos a pH 2.5 se observó un mejor comportamiento de la biomasa de tuna con una desorción inferior al 4\%. En contraste, la biomasa de alga presentó la capacidad más baja de retención de $\mathrm{Zn}$ al presentar una desorción superior al $11 \%$.

En términos de las concentraciones finales medidas en los sobrenadantes ácidos remanentes se puede afirmar que las algas presentan una menor capacidad de

Tabla 6. Concentración de metales liberados de las cenizas resultantes de la calcinación en las soluciones finales de contacto a diferente $\mathrm{pH}$.

\begin{tabular}{|c|c|c|c|c|c|c|c|}
\hline \multirow{2}{*}{ BIOMATERIAL } & \multirow{2}{*}{$\mathrm{pH}$} & \multicolumn{3}{|c|}{ Cf (mg/L) } & \multicolumn{3}{|c|}{ \% Desorción } \\
\hline & & Cd & $\mathrm{Pb}$ & $\mathrm{Zn}$ & Cd & $\mathrm{Pb}$ & $\mathrm{Zn}$ \\
\hline A1 & 2,5 & $<0,014$ & 0,72 & 4,07 & 0,26 & $\begin{array}{r}4,2748 \\
1\end{array}$ & 11,449 \\
\hline N2 & $\begin{array}{l}2,5 \\
4,5\end{array}$ & $\begin{array}{l}<0,014 \\
<0,014\end{array}$ & $\begin{array}{c}0,52 \\
<0,26\end{array}$ & $\begin{array}{l}2,13 \\
0,29\end{array}$ & $\begin{array}{l}0,25 \\
0,25\end{array}$ & $\begin{array}{l}2,2305 \\
1,1153\end{array}$ & $\begin{array}{l}4,9569 \\
0,6749\end{array}$ \\
\hline $\mathrm{T} 2$ & $\begin{array}{l}2,5 \\
4,5\end{array}$ & $\begin{array}{l}<0,014 \\
<0,014\end{array}$ & $\begin{array}{c}0,65 \\
<0,26\end{array}$ & $\begin{array}{l}1,31 \\
0,01\end{array}$ & $\begin{array}{l}0,25 \\
0,25\end{array}$ & $\begin{array}{l}3,6572 \\
1,4629\end{array}$ & $\begin{array}{l}2,9131 \\
0,0222\end{array}$ \\
\hline
\end{tabular}

A1 (alga tratada con $\mathrm{NaOH}$ ), $\mathbf{N 2}$ y $\mathbf{T} 2$ (naranja y tuna tratada con $\mathrm{NaOH}$ y $\mathrm{CaCl}_{2}$ ). 


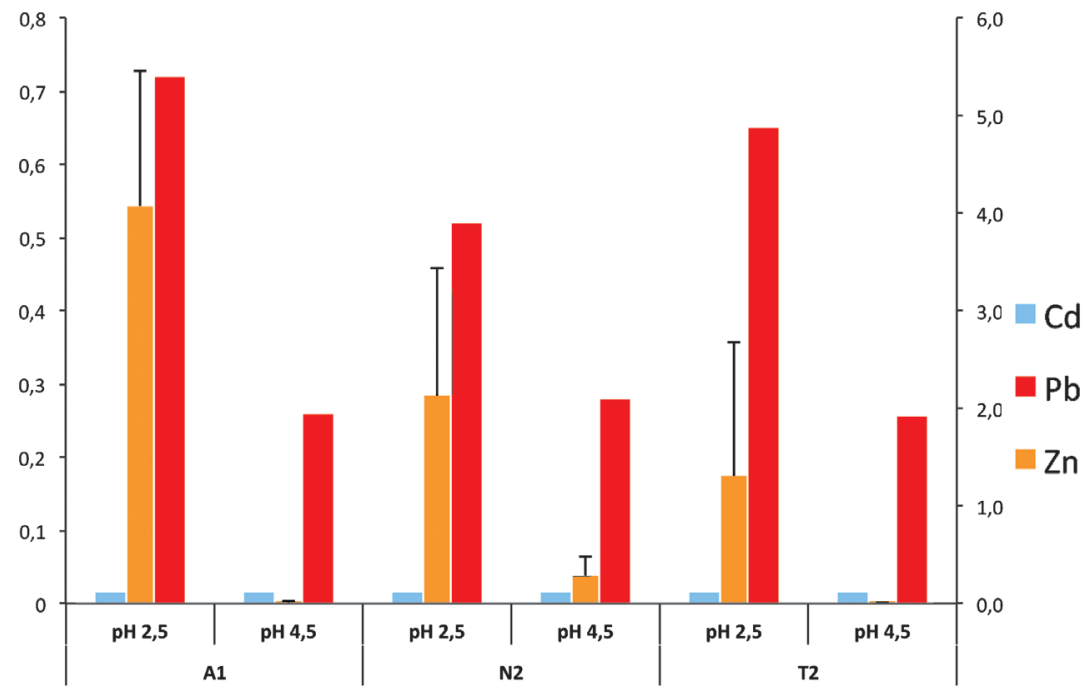

Figura 6. Desorción de Cd, Pb Zn de cenizas de algas, naranja y tuna al emplear soluciones ácidas de pH 2.5 y 4.5.

encapsulamiento de $\mathrm{Pb}$ y $\mathrm{Zn}$, lo cual puede indicar que la temperatura de calcinación empleada no es lo suficientemente alta para lograr la estabilización del residuo biológico generado. Estos resultados no concuerdan con lo obtenido por Díaz et al [5], quienes calcinaron biomasa del alga Sargassum sp. a $700{ }^{\circ} \mathrm{C}$, lixiviaron con soluciones con $\mathrm{pH}$ entre 1.5 y 7 y concluyeron que al calcinar a dicha temperatura se garantiza la estabilidad de los iones metálicos dentro de la matriz biológica utilizada como material de remoción.

En el caso de las cenizas de naranjas y tunas se presentaron las menores desorciones, a pesar de que las biomasas no se calcinaron totalmente a la temperatura y tiempo ensayados.

Tal y como se observa en la gráfica, las concentraciones desorbidas de $\mathrm{Cd}$ y $\mathrm{Pb}$ son muy bajas y similares, razón por la cual el error reportado es mínimo. En contraste, los valores reportados para $\mathrm{Zn}$ presentaron una mayor variación, que es más visible debido a que las concentraciones iniciales de este metal en las soluciones, y por consiguiente en la biomasa residual, son notablemente mayores.

Los resultados anteriores muestran que un amplio porcentaje de los metales pesados tratados permanece en la ceniza y sólo un pequeño porcentaje de los mismos es liberado por efecto del ácido nítrico empleado para desorber las cenizas. Esto indica que a $700^{\circ} \mathrm{C}$ es posible estabilizar las biomasas y disminuir la liberación de los iones metálicos removidos de la solución. Así mismo, y con el objeto de impedir que los metales se escapen de la matriz sorbente, es recomendable calcinar la biomasa de cáscara de naranja y tuna a una temperatura superior a la ensayada.

\section{CONCLUSIONES}

En los ensayos de pretratamiento se obtuvo una mejor eficiencia de sorción al emplear biomasa 
modificada $A 1, N 2$ y $T 2$, siendo este proceso más influyente en el caso del Zn. Así mismo, se observó que las biomasas de algas rojas, Citrus $s p$ y Opuntia $s p$ tienen la capacidad de remover porcentajes superiores al $90 \%$ de $\mathrm{Cd}$ y $\mathrm{Pb}$ sin requerir modificación química, mientras que su capacidad para eliminar $\mathrm{Zn}$ se encuentra por debajo del $50 \%$ en ausencia de pretratamiento.

Comparando los resultados de los ensayos en continuo frente a los ensayos batch, se puede afirmar que la capacidad de adsorción de Cd, $\mathrm{Pb}$ y $\mathrm{Zn}$ a pH 4.5 resultó mayor cuando la dosis de biomasa utilizada fue menor ( $45 \mathrm{~g}$ en continuo frente a $5 \mathrm{~g}$ en batch). Se esperaría que a mayor dosis de biomasa fuera mayor el número de sitios activos disponibles para la biosorción. Sin embargo, esto no se cumple debido a que una menor concentración de biomasa en solución permite una mejor dispersión de la misma, lo cual facilita el contacto de los grupos activos de cada uno de los materiales biológicos con el sorbato metálico, lo que produce una mayor captura de iones metálicos [2].

Los porcentajes de metal retenido por la ceniza son superiores al $95 \%$. En todos los casos, el Zn presenta el mayor porcentaje de desorción a pH $2.5(8.47 \%)$ y el más bajo a pH $4.5(0.02 \%)$. Así mismo, se observa que en todos los ensayos las concentraciones finales de $\mathrm{Cd}$ son inferiores a $0.014 \mathrm{mg} / \mathrm{L}$, que es el límite mínimo de detección de dicho metal para el espectrofotómetro utilizado para la medición de tales concentraciones.

Los ensayos de calcinación y estabilidad de las cenizas indican que las algas presentan una menor capacidad de encapsulamiento de $\mathrm{Pb}$ y $\mathrm{Zn}$, lo cual puede indicar que la temperatura de calcinación empleada no es lo suficientemente alta para lograr la estabilización del residuo biológico generado. En el caso de las cenizas de naranjas y tunas, se presentaron las menores desorciones para Pby Znen un rango porcentual que está entre 0.02 y $4.5 \%$, a pesar de que las biomasas no se calcinaron totalmente a la temperatura y tiempo ensayados.

\section{REFERENCIAS BIBLIOGRAFICAS}

[1] Muñoz Carpio, J.C. y Tapia Huanambal, N.J. (2007). Biosorción de plomo (II) por cáscara de naranja "Citrus cinensis" pretratada (Tesis de pregrado inédita). Universidad Nacional Mayor de San Marcos, Lima, Perú.

[2] Muñoz Torres, M.C. y Carrillo Chávez, A. (2007). Remoción de metales pesados en aguas residuales utilizando una macrófita acúatica (Eleocharis acicularis) muerta (Tesis de maestría inédita). Universidad Autónoma de Querétaro, Querétaro, México.

[3] Villanueva, H.C. y Tapia, H.N. (2005). Bioadsorción de Cu (II) por biomasas que contienen pectina. Rev. Per. Quím. Ing. Quím., 8(1), 11-15.

[4] Lugo, V., Barrera, C. y Hernández, S. (2013). Remoción de Plomo (II) presente en soluciones acuosas empleando cáscara de naranja (Citrus sp.) modificada. Universidad Autónoma del Estado de México. Recuperado de http://www. uaemex.mx/Red_Ambientales/docs/ memorias/Extenso/CA/EC/CAC-46.pdf.

[5] Díaz, A., Arias, J., Gelves, G., Maldonado, A., Laverde, D., Pedraza, J. y Escalante, H. (2003). Biosorción de $\mathrm{Fe}, \mathrm{Al}$ y $\mathrm{Mn}$ de drenajes ácidos de mina de carbón 
empleando algas marinas Sargassum sp. en procesos continuos. Revista Facultad de Ingeniería, (30), 34-48.

[6] Villanueva Huerta, C., y Tapia, N. (2007). Biosorción de cobre (II) por biomasa pretratada de cáscara de Citrus sinensis (naranja), Citrus limonium (limón) y Opuntia ficus (palmeta de nopal) (Tesis de pregrado inédita). Universidad Nacional Mayor de San Marcos, Lima, Perú.

[7] Clayton, L.y Wildeman, T. (1998). Processes contributing to the removal of manganese from mine drainage by an algal mixture. En National Meeting of the American Society for Surface Mining and Reclamation (pp. 17-22). Saint Louis, Missouri, EE.UU.

[8] Volesky, B. (1990). Biosorption of heavy metals. Boca Raton, FL, EE.UU.: CRC Press.

[9] Volesky, B. (2001). Detoxification of metalbearing effluents: biosorption for the next century. Hydrometallurgy, (59), 203-216.

[10] Pinzón Bedoya, M.L. y Vera Villamizar, L.E. (2009). Modelamiento de la cinética de bioadsorción de $\mathrm{Cr}$ (iii) usando cáscara de naranja. Dyna, 76(160), 95-106.

[11] Acosta, I., González, H., Moctezuma, M., Cárdenas, J., González, V.(2012). Remoción de Cromo (VI) en solución por la cáscara de naranja (Citrus sinensis Osbeck). Revista académica de investigación Tlatemoani. Recuperado de http://www.eumed.net/ rev/tlatemoani/09/rszgj.html.

[12] Zdnenek R. Volesky B." Biosorption of Heavy Metals (Cd, Cu, Ni, Pb, Zn) by Chemically- Reinforced Biomass of
Marine Algae". J. Chem. Tech. Biotech 62, 1995, 279-288.

[13] Tapia, N., Muñoz, J.C., Torres, D.F. y Yarango, A. (2003). Biosorción de Pb (II) por cáscara de naranja Citrus cinesis, modificada. Revista peruana de química e ingeniería química, 5(2), 48-53.

[14] Kratochvil, D. and Volesky, B. Advances in biosorption of heavy metals. Trends in Biotechnology, 1998, vol. 16, p. 291-300.

[15] Plaza Cazón, J. (2012). Remoción de metales pesados empleando algas Marinas (Tesis doctoral inédita). Universidad Nacional de La Plata, La Plata, Argentina.

[16] Vieira, M. G. A.; Oisiovici, R. M.; Gimenes, M. L., Silva, M. G. C. 2008. Biosorption of chromium(VI) using a Sargassum sp. packed-bed column. Bioresource Technology, 99(8), 3094-3099.

[17] Agouborde Manosalva, L. (2008). Remoción de metales pesados por medio de adsorbentes no convencionales (Tesis de maestría inédita). Universidad De La Frontera, Temuco, Chile.

[18] González N, R. (2013) Aprovechamiento de desechos plásticos, agrícolas y de crustáceos para preparar adsorbentes económicos para metales pesados. Trabajo de ingreso a la Comisión de Especialidad de Ingeniería Química. Academia de ingeniería. Guadalajara, Jalisco.

[19] Kastenmayer, P. (1995). Análisis de minerales y elementos traza en alimentos. En C. Morón, I. Zacarías, S. 
de Pablo (Eds.) Producción y manejo de datos y disposición química de alimentos en nutrición. Organización de las Naciones Unidas para la alimentación y la agricultura FAO. Recuperado de http:// www.fao.org/docrep/010/ah833s/ ah833s22.htm.

[20] Brenes Pereira, M. (2006). Utilización de cenizas en el tratamiento de las aguas residuales procedentes del beneficio de café de Coopevictoria R. L. (Tesis de pregrado inédita). Instituto Tecnológico de Costa Rica, Cartago, Costa Rica.

[21] Carrillo, F., Galindo, A., Soria, M. de J., Pérez, P., Aguilar, M., y Salinas, E. (2012). Remoción de cobre y plata en solución empleando cenizas volantes. En Pruebas preliminares. XXI Congreso Internacional de Metalurgia Extractiva. Universidad Autónoma Metropolitana, Ciudad de México.

[22] Gil Pavas, E., Saldarriaga Molina, C. y Ocampo González, A. (2001). Uso de cenizas volantes en la eliminación de $\mathrm{Cr}$ (III) de aguas residuales. Departamento de Ingeniería Química. Universidad de Antioquia, Medellín (pp. 207-212). Recuperado de http://www.icp.csic.es/ cyted/Monografias/Monografias2001/ B1-207.pdf.

[23] Skousen, J. y Ziemkiewicz, P. (2005). Performance of 116 passive treatment systems for acid drainage. National Meeting of the American Society of Mining and Reclamation. Breckenridge, CO. EE.UU.

[24] Nordstrom D.K. y Alpers C.N. (1999). Geochemistry of acid mine waters. En G.S. Plumlee, M.J. Logsdon (Eds), The environmental geochemistry of mineral deposits (pp. 133-156). Littleton, CO, EE.UU.: Reviews in Economic Geology, Society of Economic Geologists.

[25] Dionisio Ruiz Elena. Aprovechamiento de residuos vegetales para la eliminación de cobre presente en medios acuosos mediante biosorción. Universidad de Granada. Departamento de Ingeniería Química. Granada, 2010.

[26] Rodríguez, M.E., Miranda, R.C., Olivas, R. y Sosa, C. (2008). Efectos de las Condiciones de Operación Sobre la Biosorción de Pb2+, $\mathrm{Cd} 2+$ y $\mathrm{Cr} 3+$. Información Tecnológica, 19(6), 47-55.

[27] Larenas Uría, C., Andrango, D. e Inga, P. (2008). Estudio isotérmico de biosorción de plomo en aguas utilizando residuos vegetales. Quito, Ecuador: Centro de Investigación para la Valoración de la Biodiversidad (CIVABI), Universidad Politécnica Salesiana.

[28] Campos Medina, E. (2003). Estudio de la adsorción de cromo hexavalente como biomaterial la ectodermis de opuntia. Quivera, 10(1), 16-31.

[29] Inga Manchola. P.A. y Larenas Uria, C.F. (2012) Estudio Isotérmico de biosorción de cadmio, cromo, plomo y zinc en solución acuosa empleando el pinzote (raquis) de plátano (Musa paradisiaca L.). Quito, Ecuador: Universidad politécnica salesiana.

[30] López, F.A., Martín, M.l., Pérez, C., López, A. y Alguacil, F.J. (2003). Adsorción de metales pesados sobre cascarilla de laminación. Revista de Metalurgia de Madrid, 39, 215-223. 
[31] Mori, C M., Maldonado, G H.., Guzmán, L E.., Eyras, CC.. Bernardelli, M. Viera, y E. Donati. (2013). Estudio cinético e isotérmico de la biosorción de zinc (II) y cadmio (II) para un sistema monometálico-bimetálico por Undaria pinnatifida sp. Revista peruana de química e ingeniería química. 16(2), 39-46.

[32] Aguilar, D., Cortes, R. (2010) Uso de residuos de naranja (Citrus sinensis) y tamarindo (Tamarindus indica) como biosorbentes en la remocion de plomo, cadmio y zinc de aguas contaminadas (Tesis de pregrado inédita). Universidad
Michoacana de San Nicolas de Hidalgo, Morelia, México.

[33] Cuizano, A., Reyes, U., Domínguez, S., Llanos, B., Navarro, A. (2010). Relevancia del $\mathrm{PH}$ en la adsorción de iones metálicos mediante algas pardas. Revista de la Sociedad Química Perú, 76(2), 123-130.

[34] Navarro, A.; Collado, D. y Font, X.; Movilidad de los metales pesados en el acuífero deltaico del río Almanzora (Almería), Tecnología del agua, 172, pp. 33-45 (1998). 